\title{
PRECISION MEASUREMENTS AT A MUON COLLIDER
}

\author{
S. Dawson \\ Physics Department \\ Brookhaven National Laboratory \\ Upton, New York 11973
}

\begin{abstract}
We discuss the potential for making precision measurements of $M_{W}$ and $M_{T}$ at a muon collider and the motivations for each measurement. A comparison is made with the precision measurements expected at other facilities. The measurement of the top quark decay width is also discussed.
\end{abstract}

\section{INTRODUCTION}

A $\mu^{+} \mu^{-}$collider with high luminosity and narrow beam spread offers the possibility of performing high precision measurements of fundamental masses and decay widths occurring in the Standard Model and in some extensions of the Standard Model. We discuss precision measurements of the $W$ mass and the top quark mass and width. Measurements of the Higgs boson mass and width, both in the Standard Model and in SUSY models are discussed in Ref. [1]. We pay particular attention to the motivation for making each precision measurement and the experimental precision which is necessary in order to test the theoretical consistency of the Standard Model or to verify the existence of new physics.

Each of these precision measurements depends on knowing the relevant energy and building a storage ring to maximize the luminosity at that energy. The mass measurements of the $W$ and top quark are made by scanning the threshold energy dependences of the cross sections. The threshold energy dependences will be smeared by radiation from the initial state particles, limiting the precision of the measurements. Because the muon is much heavier than the electron, there will be less initial state radiation and the beam energy resolution may be better in a muon collider than in an electron collider, leading 
to the possibility of more precise measurements.

\section{MEASUREMENT OF THE $W$ MASS}

A precision measurement of the $W$ mass is of fundamental importance to our understanding of the Standard Model. Combined with a precision measurement of the top quark mass, the consistency of the Standard Model can be checked since the $W$ mass is predicted as a function of the top quark mass.

The current world average on the $W$ mass is obtained by combining data from UA2, CDF, and D0: [2]

$$
M_{W}=80.23 \pm .18 \mathrm{GeV} .
$$

With more data from CDF and D0, both the systematic and statistical errors will decrease and it has been estimated [2] that with $100 \mathrm{pb}^{-1}$ it will be possible to obtain:

$$
\Delta M_{W}^{\text {Tevatron }} \sim 110 \pm 20 \mathrm{MeV}
$$

while $1000 p b^{-1}$ will give

$$
\Delta M_{W}^{\text {Tevatron }} \sim 50 \pm 20 \mathrm{MeV},
$$

where the first error is statistical and the second is systematic.

At LEP-II, the error on $M_{W}$ can be reduced still further. There are two general strategies for obtaining a mass measurement. The first is to reconstruct the decay products of the $W$, while the second method is to measure the excitation curve of the $W$ pair production cross section as the energy is varied. Both methods give approximately the same precision. By reconstructing the $W$ decay products with $500 \mathrm{pb}^{-1}$ (3 years running) at $\sqrt{s}=190 \mathrm{MeV}[2]$,

$$
\Delta M_{W}^{\mathrm{LEP}-\mathrm{II}} \sim 40 \mathrm{MeV} .
$$

The precision is ultimately limited by the knowledge of the beam energy, $\Delta E^{\text {beam }} \sim 20 \mathrm{MeV}$.

It is possible that a muon collider could obtain a more precise measurement of $M_{W}$ than is possible at LEP-II. We will discuss the design restrictions on a muon collider in order to make this the case. The beam spread at a lepton collider can be roughly assumed to have a Gaussian energy resolution with a rms deviation, $[1]$

$$
\delta \sim 60 \mathrm{MeV}\left(\frac{R}{.06 \%}\right)\left(\frac{\sqrt{s}}{2 M_{W}}\right)
$$

leading to an energy resolution smaller that the $W$ decay width. A typical parameter for a high energy $e^{+} e^{-}$collider is $R=1$, while a lower value is 
envisioned for a muon collider, (say $R \sim .06 \%$ ), due to the fact that a muon collider will have less initial state radiation (ISR) than an $e^{+} e^{-}$collider. Here we investigate the requirements on $R$ in order for a measurement of $M_{W}$ to be made at a muon collider which will improve on the precision expected at LEP-II.

The procedure is to study the shape of the $l^{+} l^{-} \rightarrow W^{+} W^{-}$cross section as a function of the center-of-mass energy, $\sqrt{s}$, and to fit a theoretical expectation to the cross section. The many theoretical effects which must be included are discussed by Stirling [3] and we follow his discussion closely.

We compute the cross section for off-shell $W$ pair production including Coulomb effects as, $[3]$

$$
\sigma_{a}(s)\left(l^{+} l^{-} \rightarrow W^{+} W^{-}\right)=\left[1+\delta_{C}(s)\right] \int d s_{1} \int d s_{2} \rho\left(s_{1}\right) \rho\left(s_{2}\right) \sigma_{0}(s, s 1, s 2),
$$

where

$$
\rho(s)=\frac{\Gamma_{W}}{\pi M_{W}}\left[\frac{s}{\left(s-M_{W}^{2}\right)^{2}+s^{2} \Gamma_{W}^{2} / M_{W}^{2}}\right]
$$

and $\sigma_{0}\left(s, s_{1}, s_{2}\right)$ is the Born cross section for producing a $W^{+} W^{-}$pair with $W^{ \pm}$energies $\sqrt{s_{1}}$ and $\sqrt{s_{2}}$. (Electroweak radiative corrections are negligible in the threshold region.) This procedure defines what we mean by the $W$ mass and is in fact the same definition as used in LEP measurements. The Coulomb corrections are included in the factor $\delta_{C}(s)$ and arise from the fact the $W^{+} W^{-}$ cross section diverges as $1 / v$ at threshold. The analytic expression for $\delta_{C}(s)$ can be found in Ref. [4].

Finally, the corrections due to initial state radiation, which are sensitive to the lepton mass, $m_{l}$, must be included. The ISR is the first correction which differentiates between an electron and a muon collider. These corrections are included with a radiator function $F(x, s)$ (given in Ref. [5]) to obtain our final result for the $W^{+} W^{-}$pair production cross section:

$$
\sigma(s)\left(l^{+} l^{-} \rightarrow W^{+} W^{-}\right)=\frac{1}{s} \int d s^{\prime} F(x, s) \sigma_{a}\left(s^{\prime}\right)
$$

where $x \equiv 1-\frac{s^{\prime}}{s}$ and

$$
F(x, s) \sim t x^{t-1}+\left(\frac{x}{2}-1\right) t+\ldots
$$

with $t=\frac{2 \alpha}{\pi}\left[\log \left(\frac{s}{m_{l}^{2}}\right)-1\right]$. We see that the ISR is potentially much larger at an $e^{+} e^{-}$machine than at a muon collider. The various contributions to $l^{+} l^{-} \rightarrow W^{+} W^{-}$are shown in Fig. 1. Near threshold, $\sqrt{s} \sim 2 M_{W}$, the cross section rises rapidly with $\sqrt{s}$. As expected, there is less reduction of the cross section due to ISR at a muon collider than at an electron collider. In Fig. 
2 , we see that the cross section is very sensitive to the precise value of $M_{W}$ assumed. It is straightforward to find the statistical error for a given efficiency, $\epsilon$, and luminosity, $\int \mathcal{L},[3]$

$$
\Delta M_{W}^{\text {stat }}=\frac{1}{\left|\frac{d \sigma}{d M}\right|} \sqrt{\frac{\sigma}{\epsilon \int \mathcal{L}}} .
$$

From Fig. 3, we find that the minimum statistical error occurs at $\sqrt{s} \sim$ $2 M_{W}$ (where the cross section has the steepest dependance on energy). The statistical error is obviously not much different at a muon collider than at an electron collider, so our results correspond to those of Ref. [3]. That this must be the case can be seen from Fig. 1; at threshold, the effects of initial state radiation are small. At the minimum:

$$
\Delta M_{W}^{\text {stat }} \sim 90 \mathrm{MeV}\left[\frac{\epsilon \int \mathcal{L}}{100 p b^{-1}}\right]^{-1 / 2} .
$$

If it were possible to have $1 \mathrm{fb}^{-1}$ concentrated at $\sqrt{s} \sim 2 M_{W}$ with an efficiency $\epsilon=.5$, then a muon collider could find $\Delta M_{W}^{\text {stat }} \sim 40 \mathrm{MeV}$ ! Unfortunately, the luminosity of a muon collider decreases rapidly away from the design energy, so $1 \mathrm{fb}^{-1}$ at $\sqrt{s} \sim 160 \mathrm{GeV}$ is probably an unrealistic goal.

It is also necessary to consider the systematic error, which is primarily due to the uncertainty in the beam energy. From Fig. 2, changing the beam energy is equivalent to a shift in $M_{W}, \Delta M_{W}^{\text {sys }} \sim \Delta E^{\text {beam }}$. Therefore, to obtain a measurement with the same precision as LEP-II, a muon collider must be designed with $\Delta E^{\text {beam }} \sim 20 \mathrm{MeV}$, which corresponds to $R \sim .02 \%$.

The bottom line is that a $\mu^{+} \mu^{-}$collider must have on the order of $1 \mathrm{fb}^{-1}$ at $\sqrt{s} \sim 2 M_{W}$ and an extremely narrow beam spread, $R \sim .02 \%$, in order to be competitive with LEP-II for a measurement of $\Delta M_{W}$.

\section{PRECISION MEASUREMENT OF THE TOP QUARK MASS AND WIDTH}

A muon collider will also be able to obtain a very precise measurement of the top quark mass, as has been discussed in detail by Berger.[6] Here, we concentrate on the physics motivations for making a precision measurement of the top quark mass.

Since at the moment we have no firm prediction for the top quark mass, a precision measurement of $M_{T}$ is not particularly interesting in itself. However, when combined with a precision measurement of $M_{W}$, it tests the consistency of the Standard Model. This is because the prediction for $M_{W}$ in the Standard 
Model depends on $M_{T},[7]$

$$
M_{W}^{2}=M_{Z}^{2}\left[1-\frac{\pi \alpha}{\sqrt{2} G_{\mu} M_{w}^{2}(1-\Delta r)}\right]^{\frac{1}{2}}
$$

with $\Delta r \sim \frac{M_{T}^{2}}{M_{W}^{2}},(\Delta r$ also depends logarithmically on the Higgs boson mass). In Figure 4, we show the relationship between $M_{T}$ and $M_{W}$ in the Standard Model (where we have assumed $M_{H}=100 \mathrm{GeV}$ and included only contributions to $\Delta r$ which depend quadratically on the top quark mass). For $M_{T}=175 \mathrm{GeV}$, a measurement of $M_{W}$ to $\Delta M_{W}=40 \mathrm{MeV}$ requires a measurement of $M_{T}$ to $\Delta M_{T}=6 \mathrm{GeV}$ in order to check the consistency of the Standard Model, while $\Delta M_{W}=20 \mathrm{MeV}$ requires $\Delta M_{T}=3 \mathrm{GeV}$. Given the expected precision on $\Delta M_{W}$ at LEP-II, it is clear that there is no motivation for a more precise measurement of $M_{T}$ than several GeV.

A precise measurement of $M_{T}$ and $M_{W}$ also gives some information on the Higgs mass. For example, if $\Delta M_{W}=40 \mathrm{MeV}, \Delta M_{T}=4 \mathrm{GeV}$ and the true value of $M_{H}$ were $100 \mathrm{GeV}$, then one could deduce from the electroweak measurements that at the $1 \sigma$ level, $50<M_{H}<200 \mathrm{GeV}$.[2]

From the Tevatron, we will have $\Delta M_{T} \sim 8 \mathrm{GeV}$ with $100 p b^{-1}$ and $\Delta M_{T} \sim$ $\pm 4 \mathrm{GeV}$ with $1000 p b^{-1}$.[2] The LHC experiments are designed such that with 1 year of running, $\int \mathcal{L}=10 \mathrm{fb}^{-1}$, a value on the order of, $\Delta M_{T} \sim 3 \mathrm{GeV}$ will be obtained.[2] In contrast, the values which would be obtained from a muon collider [6]

$$
\Delta M_{T}^{\mu^{+} \mu^{-}} \sim 300 \mathrm{MeV}
$$

and from an electron collider [8]

$$
\Delta M_{T}^{e^{+} e^{-}} \sim 520 \mathrm{MeV}
$$

are considerably more precise.

A precision measurement of the top quark width conveys significantly more information than a precision measurement of the mass. This is because the width is sensitive through loop effects to new particles contained in extensions of the Standard Model. Single top production at the Tevatron will measure the top quark width to roughly, [9]

$$
\frac{\Delta \Gamma_{T}^{\text {Tevatron }}}{\Gamma_{T}} \sim .3
$$

while a $500 \mathrm{GeV} e^{+} e^{-}$collider might obtain[8]

$$
\frac{\Delta \Gamma_{T}^{e^{+} e^{-}}}{\Gamma_{T}} \sim .2
$$


Presumably, a $\mu^{+} \mu^{-}$collider will do even better. Such measurements will be capable of limiting the low mass particle spectrum of supersymmetric models and it would be interesting to have a systematic comparison of the capabilities of an $e^{+} e^{-}$and $\mu^{+} \mu^{-}$collider and the corresponding limits on SUSY particles.

\section{REFERENCES}

1. See, for example, V. Barger, M. Berger, J. Gunion, and T. Han, Phys. Rev. Lett. 75 (1995) 1462, hep-ph/9504330; V. Barger, contribution to this workshop.

2. F. Merritt, H. Montgomery, A. Sirlin, and M. Swartz, Precision Tests of Electroweak Physics, report of the DPF Committee on Long Term Planning, 1994.

3. W. Stirling, DTP-95-24 (1995), hep-ph/9503320.

4. V. Fadin, V. Khoze, A. Martin, and W. Stirling, Phys. Lett. B363 (1995) 112, hep-ph/9507422.

5. F. Berends, in $Z$ Physics at LEP I, CERN Yellow Report No. 89-08, Geneva, 1989, Vol. 1, edited by G. Altarelli, R. Kleiss, and C. Verzegnassi.

6. M. Berger, presented at International Symposium on Particle Theory and Phenomenology, Ames, Iowa (1995), hep-ph/9508209.

7. W. Marciano, Ann. Rev. Nucl. Part. Sci 41 (1991) 469.

8. P. Igo-Komines, in Proceedings of the Workshop on Physics and Linear $e^{+} e^{-}$Colliders, Waikoloa, Hawaii, 1993, edited by F. Harris, S. Olsen, S. Pakvasa, and X. Tata (World Scientific, Singapore, 1993); K. Fujii, T. Matsui, and Y. Sumino, Phys. Rev. D50 (1994) 4341.

9. D. Carlson and C.P. Yuan, presented at International Symposium on Particle Theory and Phenomenology, Ames, Iowa (1995), hep-ph/9509208. 


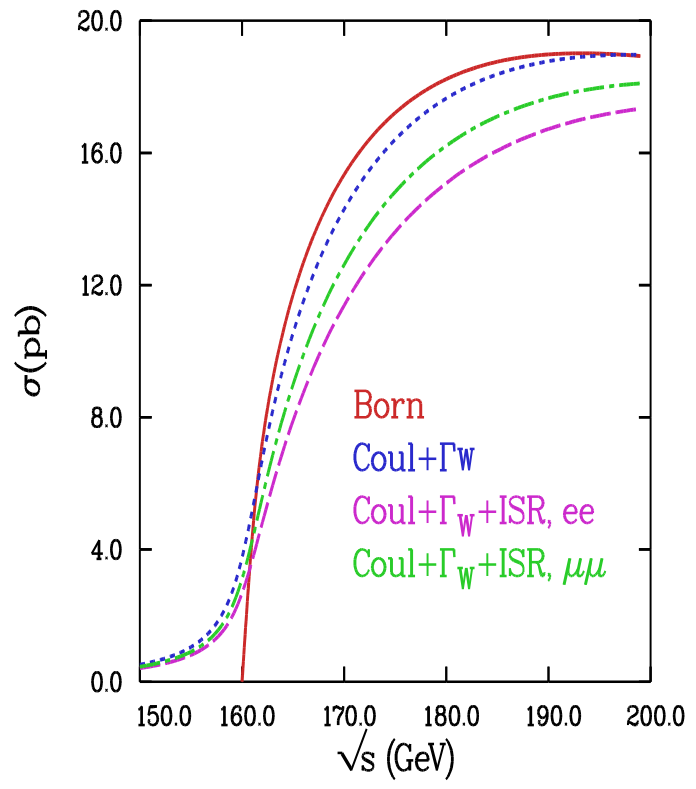

Figure 1: Contributions to the $l^{+} l^{-} \rightarrow W^{+} W^{-}$cross section. The solid curve includes only the tree level cross section, while the dotted curve includes the Coulomb and finite $W$ width effects. The long-dashed and dot-dashed curves include the effects of ISR for $e^{+} e^{-}$and $\mu^{+} \mu^{-}$colliders, respectively. 


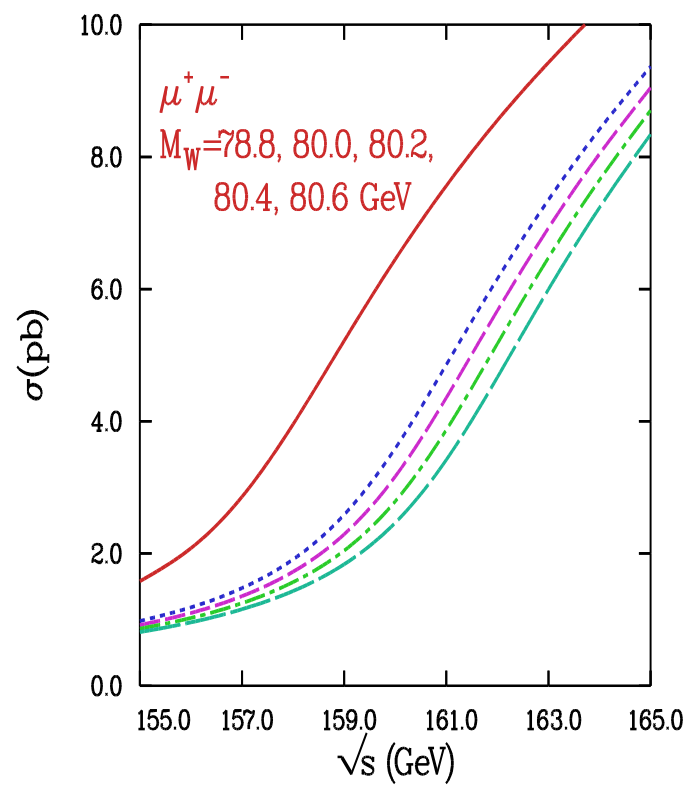

Figure 2: Cross section for $\mu^{+} \mu^{-} \rightarrow W^{+} W^{-}$. The solid curve has $M_{W}=$ $78.8 \mathrm{GeV}$. 


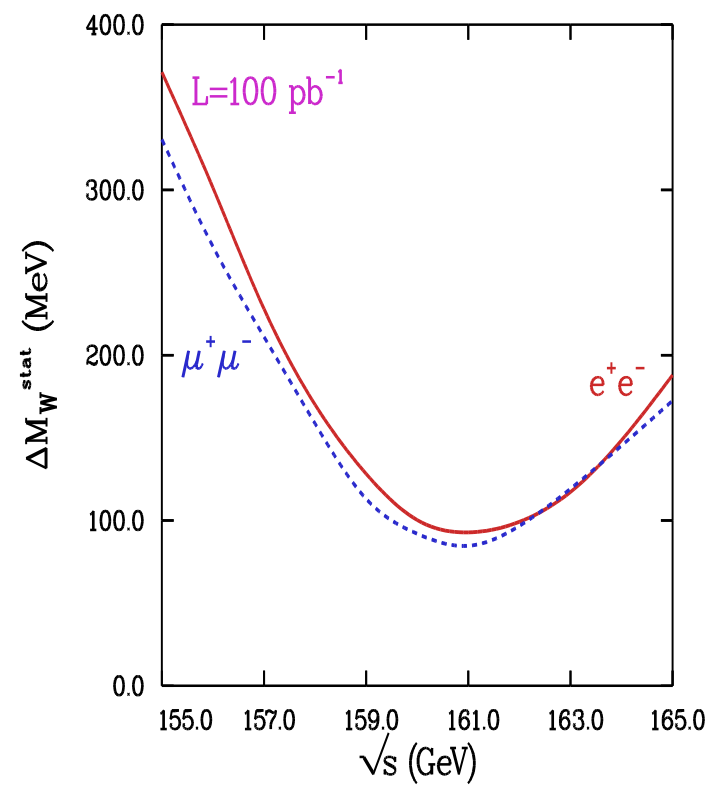

Figure 3: Statistical error on $M_{W}$ from $l^{+} l^{-} \rightarrow W^{+} W^{-}$from an absolute measurement of the rate with an integrated luminosity, $\mathcal{L}=100 p b^{-1}$. 


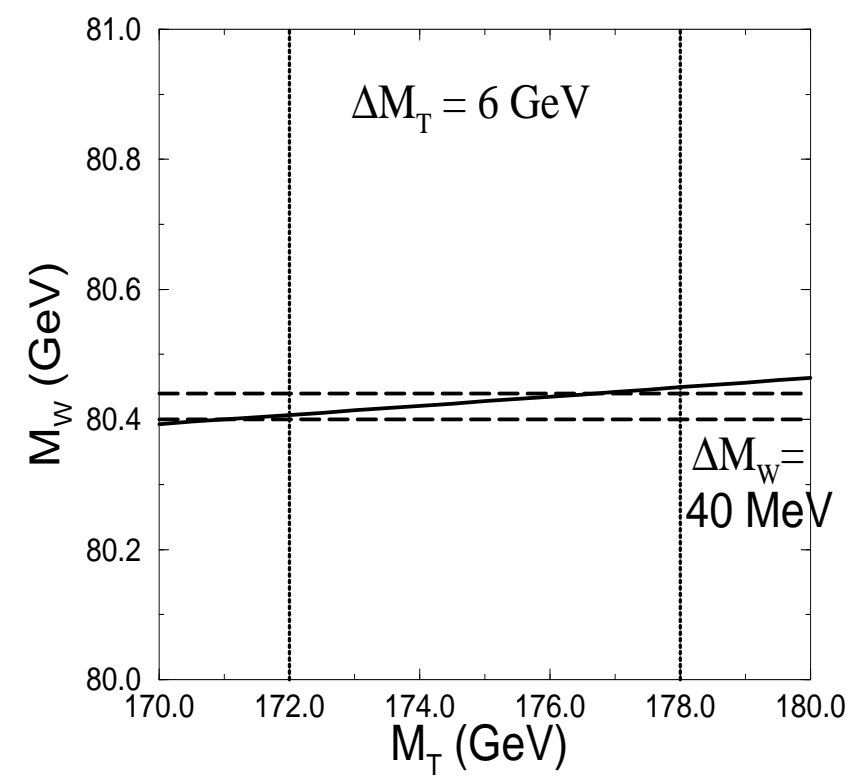

Figure 4: Dependence of the predicted $W$ mass on the top quark mass in the Standard Model. 